\title{
Gastric outlet obstruction caused by metastatic tumor of the stomach originating from primary breast cancer: A case report
}

\author{
MAHO OGAWA $^{1}$, TSUTOMU NAMIKAWA ${ }^{1}$, TOYOKAZU OKI ${ }^{1}$, JUN IWABU $^{1}$, MASAYA MUNEKAGE $^{1}$, \\ HIROMICHI MAEDA ${ }^{2}$, TAKAHIKO TAMURA ${ }^{3}$, TOMOAKI YATABE ${ }^{3}$, HIROYUKI KITAGAWA $^{1}$, \\ KEN DABANAKA $^{1}$, TAKEKI SUGIMOTO ${ }^{1}$, MICHIYA KOBAYASHI ${ }^{2,4}$ and KAZUHIRO HANAZAKI ${ }^{1}$ \\ ${ }^{1}$ Department of Surgery, Kochi Medical School; ${ }^{2}$ Cancer Treatment Center, Kochi Medical School Hospital; \\ Departments of ${ }^{3}$ Anesthesiology and Intensive Care Medicine, and ${ }^{4}$ Human Health and \\ Medical Sciences, Kochi Medical School, Nankoku, Kochi 783-8505, Japan
}

Received July 25, 2018; Accepted September 17, 2018

DOI: $10.3892 / \mathrm{mco} .2018 .1722$

\begin{abstract}
The most common sites of breast cancer metastasis are the bone, liver, lung and brain, while gastrointestinal metastasis from breast cancer is rare. We herein present the case of a 68-year-old woman who was admitted to our department with nausea and appetite loss. The patient's medical history included right mastectomy with sentinel lymph node biopsy 5 years earlier for invasive lobular carcinoma, measuring $6.2 \mathrm{~cm}$ in greatest diameter, without lymphovascular invasion. Two years after the surgery, the patient developed brain metastasis and underwent metastasectomy to control the neurological symptoms, including unsteadiness and asthenia. After the second surgery, the patient received systemic chemotherapy using $\mathrm{S}-1$, followed by bevacizumab plus paclitaxel. However, due to bevacizumab-related cardiotoxicity, the treatment was switched to eribulin. On esophagogastroduodenoscopy, an elevated lesion was identified in the antrum, causing severe narrowing of the gastric outlet. Biopsy and histological examination of the tumor revealed infiltration of the gastric wall by undifferentiated neoplastic cells with poor adhesion, morphologically similar to invasive lobular carcinoma, and immunohistochemical staining was positive for estrogen receptor, mammaglobin and GATA3. Finally, ${ }^{18}$ F-2-deoxy-2-fluoro-D-glucose (FDG) positron emission tomography combined with computed tomography imaging revealed FDG uptake across the thickness of the antral wall. The patient was diagnosed with gastric metastasis from the original breast cancer and subsequently underwent endoscopic self-expandable metallic stent (SEMS) placement. There were no procedure-related adverse events, and the patient remained
\end{abstract}

Correspondence to: Dr Tsutomu Namikawa, Department of Surgery, Kochi Medical School, Kohasu, Oko-cho, Nankoku, Kochi 783-8505, Japan

E-mail: tsutomun@kochi-u.ac.jp

Key words: breast cancer, metastatic gastric tumor, lobular carcinoma, gastric outlet obstruction alive under best supportive care 4 months after SEMS placement. To the best of our knowledge, this is the first reported case of gastric outlet obstruction caused by metastatic breast carcinoma managed by SEMS placement. While such a diagnosis is rare, clinicians treating patients with gastric metastases should be aware of possible gastric outlet obstruction and SEMS placement as an effective palliative intervention.

\section{Introduction}

Breast cancer is the most common malignancy among women worldwide. Among breast cancer subtypes, invasive ductal carcinoma is the one most commonly associated with metastasis (1). Invasive lobular cancer is the second most common invasive type of breast cancer, and is associated with a heterogeneous group of histologically different types of metastatic spread and potentially uncommon patterns of metastatic site involvement $(1,2)$. The most common sites for breast cancer metastases are the bone, liver, lung and brain, while gastrointestinal metastasis from breast cancer is rare. However, acknowledging this entity is important for accurate and timely diagnosis and treatment $(2,3)$.

We herein report the case of a patient with a metastatic gastric tumor arising from invasive lobular carcinoma of the breast, complicated by gastric outlet obstruction (GOO), which was managed by metallic stent placement.

\section{Case report}

A 68-year-old female patient presented to the Kochi Medical School Hospital (Nankoku, Japan) in March 2018 with nausea and appetite loss. The patient's medical history included right mastectomy with sentinel lymph node biopsy for right breast cancer 5 years earlier. The pathological diagnosis was invasive lobular carcinoma, $6.2 \mathrm{~cm}$ in greatest diameter, without lymphovascular invasion. Immunohistochemical examination of the tumor revealed $40 \%$ estrogen receptor positivity, $1 \%$ progesterone receptor positivity and negative staining for human epidermal growth factor receptor 2 (HER2), with a Ki-67 index of 15\%. Two years after the surgery, the patient developed brain metastasis and underwent metastasectomy 
to control neurological symptoms such as unsteadiness and asthenia. Postoperatively, the patient received systemic chemotherapy using S-1, followed by bevacizumab plus paclitaxel, although the treatment was subsequently changed to eribulin due to bevacizumab-related cardiotoxicity.

The laboratory findings on admission were as follows: Decreased red blood cell count $\left(296 \times 10^{4} / \mathrm{mm}^{3}\right.$; normal range, $386-492 \times 10^{4} / \mathrm{mm}^{3}$ ), decreased white blood cell count $\left(1.0 \times 10^{3} / \mathrm{mm}^{3}\right.$; normal range, $\left.3.3-8.6 \times 10^{3} / \mathrm{mm}^{3}\right)$ and increased C-reactive protein levels $(34.21 \mathrm{mg} / \mathrm{dl}$; normal values $<0.14 \mathrm{mg} / \mathrm{dl}$ ). On esophagogastroduodenoscopy, an elevated lesion was identified occupying the entire circumference of the antrum and causing a narrowing of the gastric outlet (Fig. 1). Biopsy of the tumor followed by histological examination revealed infiltration of the wall of the antrum by undifferentiated neoplastic cells with poor adhesion, resembling invasive lobular carcinoma (Fig. 2A); immunohistochemical staining revealed positivity for estrogen receptor (Fig. 2B), mammaglobin (Fig. 2C) and GATA3 (Fig. 2D). Imaging by ${ }^{18} \mathrm{~F}$-2-deoxy-2-fluoro-D-glucose (FDG) positron emission tomography combined with computed tomography revealed FDG uptake across the full thickness of the antral wall (Fig. 3). These findings indicated a clinical diagnosis of gastric metastasis from the primary breast cancer.

The patient eventually developed GOO that markedly worsened her quality of life; thus, placement of an endoscopic self-expandable metallic stent (SEMS) was performed to resolve the obstruction-induced clinical symptoms (Fig. 4). There were no procedure-related adverse events. The date of last contact was August 2018, and the patient remained alive under best supportive care 5 months after the procedure.

\section{Discussion}

We herein present a case of GOO caused by a metastatic tumor of the stomach originating from an invasive lobular carcinoma of the breast that was resected 5 years earlier. SEMS placement was used to relieve the obstruction, as it is an effective and safe procedure for maintaining the quality of life of patients under best supportive care. To the best of our knowledge, this is the first reported case of GOO caused by metastatic gastric tumor managed by SEMS placement.

Gastric involvement by metastatic breast cancer is a rare clinical diagnosis. Furthermore, invasive lobular breast carcinoma is less likely to involve the gastrointestinal tract compared with invasive ductal carcinoma, with the most frequent metastatic sites being the bone, gynecological organs, peritoneum and retroperitoneum (3-5). In this respect, the present case highlights the importance of considering metastatic tumor of the stomach secondary to invasive lobular carcinoma of the breast, and the importance of immunohistochemical analysis, such as staining for estrogen and progesterone receptors $(2,3,5,6)$.

GATA3 is a multifunctional transcription factor that is important for the development and function of ductal epithelial cells, including those of breast, urothelia, epidermis and skin adnexa, wherein specific nuclear proteins recognize G-A-T-A nucleotide sequences in target gene promoters (7). As the majority of primary and metastatic mammary tumors express GATA3 (positive rate of $80-90 \%$ ), it is a potentially useful

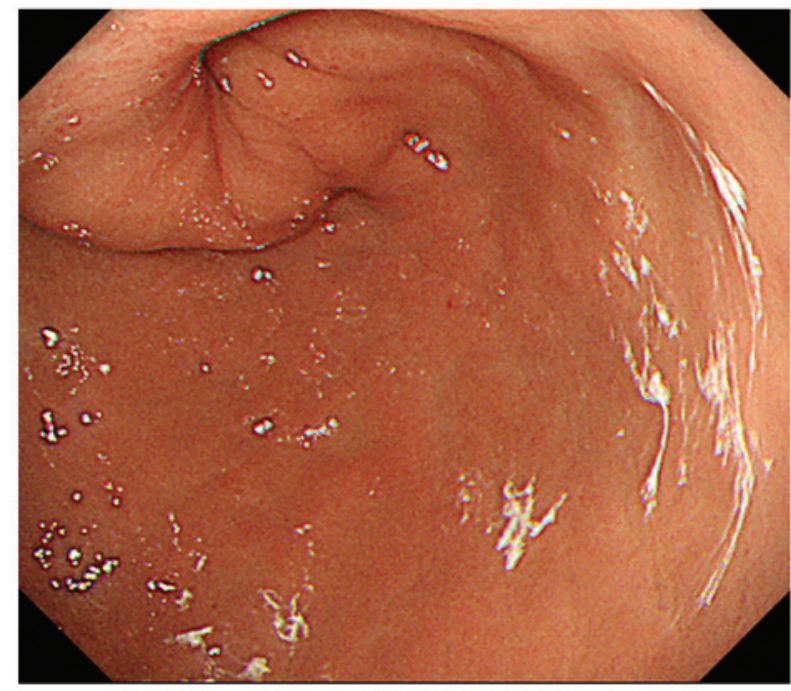

Figure 1. Esophagogastroduodenoscopy showing a circumferential elevated lesion in the antrum with superficial erosions and narrowing of the gastric outlet.

addition to hormonal markers, such as estrogen and progesterone receptors, for identifying metastatic cells of mammary origin (8). In the present case, positive immunohistochemical staining of these three markers was observed.

The endoscopic and radiological appearance resemble linitis plastica due to the diffuse infiltration of the submucosa and muscularis propria, with circumferential thickening and narrowing of the lumen, as metastatic lobular carcinoma infiltrates within the serosal, muscular and submucosal layers, with cord-like projections of small cells (6). The treatment generally recommended for gastric metastases from breast cancer is systemic chemotherapy and/or hormonal therapy $(3,4,6)$. When an unusual lesion is detected in a patient with invasive lobular carcinoma, metastatic disease should be considered in the differential diagnosis, and immunohistochemical analysis is recommended for accurate diagnosis.

Patients with malignant GOO tend to develop undesirable clinical symptoms that are detrimental to the quality of life of the patients, such as nausea, vomiting, abdominal pain and difficulty eating (9). Fluoroscopic or/and endoscopic SEMS placement as palliative treatment for malignant GOO is generally safe, easily performed and effective, and is associated with higher clinical success rates and lower morbidity and mortality rates compared with palliative surgery $(9,10)$. By contrast, Jang et al (11) reported that palliative gastrojejunostomy was significantly associated with longer overall survival and lower risk of re-intervention compared with SEMS placement in patients with malignant GOO caused by unresectable gastric cancer using a propensity score matching analysis. Overall, the choice of systemic treatment, such as chemotherapy and/or hormonal therapy, for breast cancer metastasis is based upon presenting symptoms, age and general performance status, and surgical palliation should be considered only under emergency conditions to bypass the obstruction (3). The bone marrow of the patient in the present case was exhausted due to long-term systemic chemotherapy; thus, we selected SEMS placement to avoid unfavorable complications associated with the bypass procedure. 

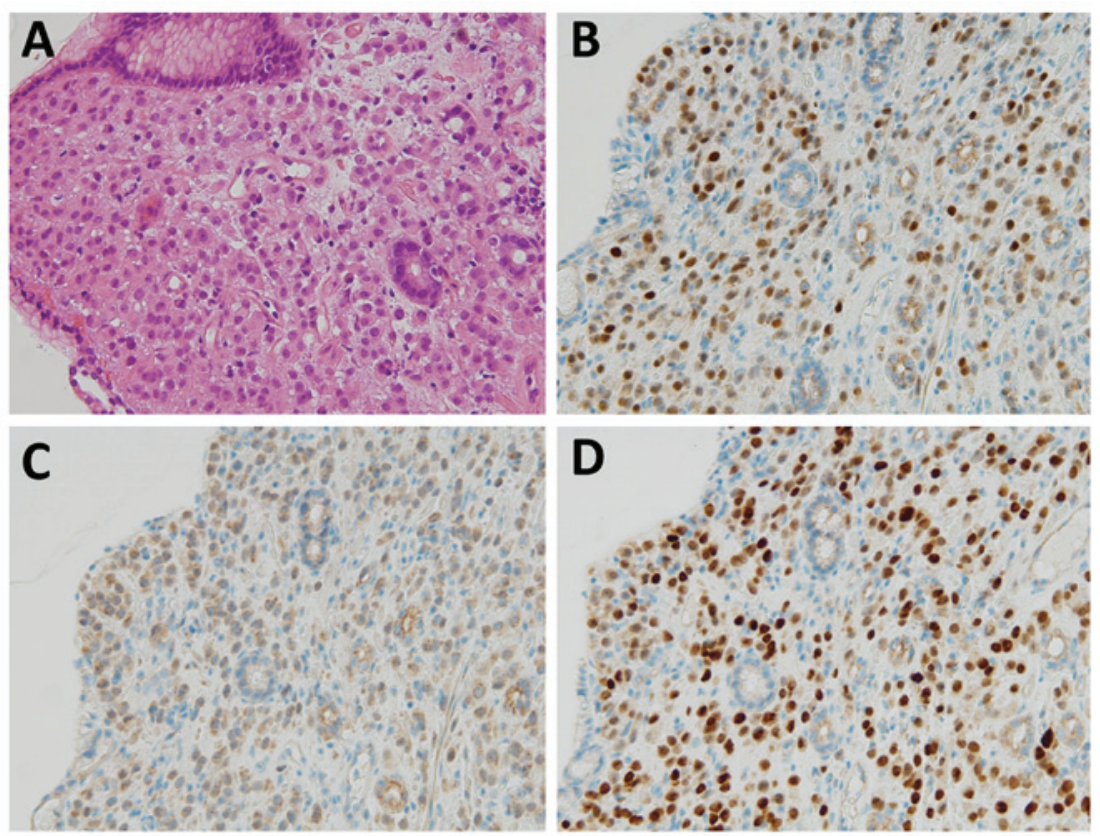

Figure 2. Histological findings from the gastric biopsy specimen. (A) Hematoxylin and eosin staining revealed infiltration by poorly differentiated adenocarcinoma cells. Immunohistochemical examination demonstrated positivity for (B) estrogen receptor, (C) mammaglobin and (D) GATA3. Hematoxylin and eosin staining; magnification, x100.

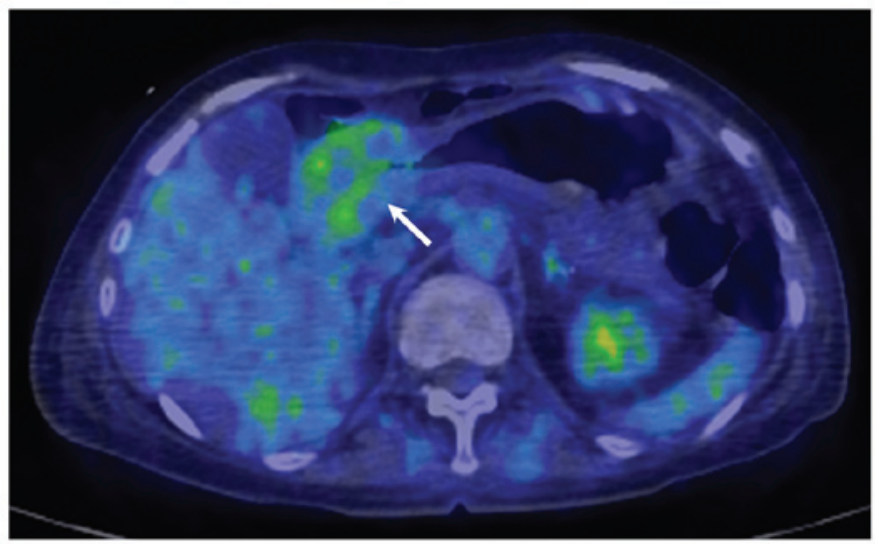

Figure $3 .{ }^{18} \mathrm{~F}$-2-deoxy-2-fluoro-D-glucose (FDG) positron emission tomography combined with computed tomography imaging revealed thickening of the entire gastric antrum wall with FDG uptake (arrow).

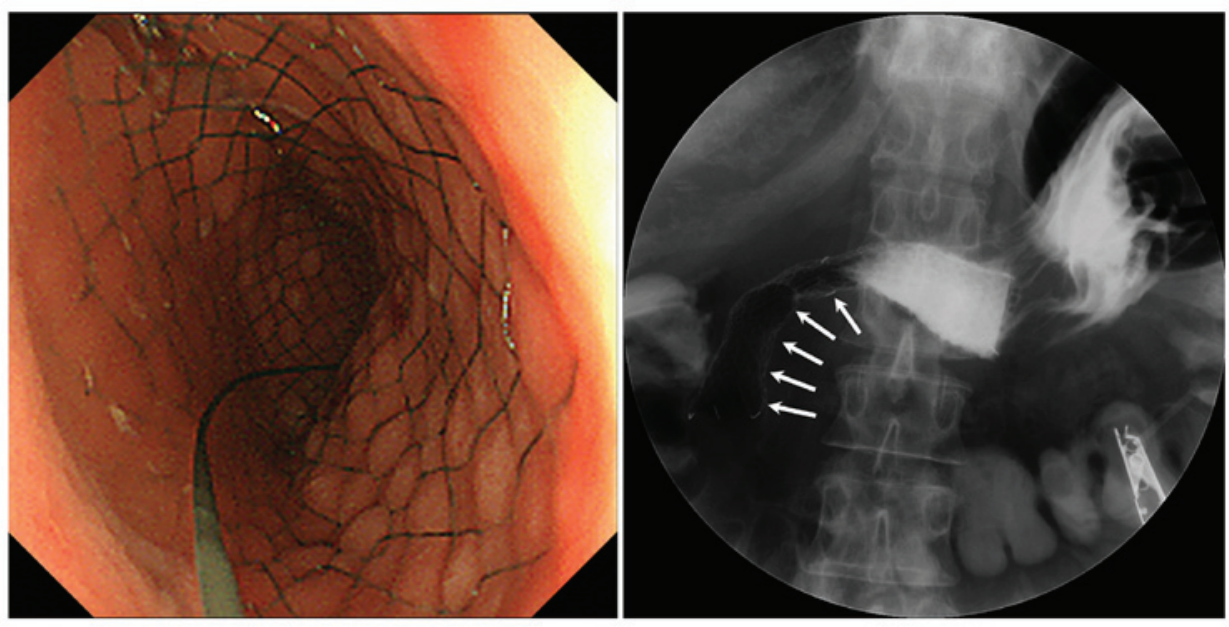

Figure 4. Endoscopic and fluoroscopic images following self-expandable metallic stent placement. (A) A self-expandable metallic stent was placed under guide-wire assisted endoscopy. (B) Fluoroscopy using water-soluble contrast medium showed opening of the gastric outlet obstruction (arrows). 
In conclusion, this case indicates that SEMS placement may be a promising approach to the management of patients with GOO caused by unresectable advanced gastric cancer as well as metastatic gastric tumors, and may contribute to improved quality of life for these patients. Further investigations with a larger accumulation of cases and/or prospective studies are required to establish the optimal treatment for GOO caused by metastatic tumors to the stomach originating from other primary malignancies.

\section{Acknowledgements}

Not applicable.

\section{Funding}

No funding was received

\section{Availability of data and materials}

Not applicable.

\section{Authors' contributions}

MO and TN contributed to the writing of the manuscript. MK and KH supervised the study. MO, TN, TO, JI, HM, TT, TY, $\mathrm{HK}, \mathrm{KD}$ and TS served as the attending physicians for the presented patient. All the authors have read and approved that final version of this manuscript.

\section{Ethics approval and consent to participate}

Not applicable.

\section{Patient consent for publication}

The patient has given consent for the publication of the case details and associated images.

\section{Competing interests}

The authors declare that they have no competing interests to disclose.

\section{References}

1. Arpino G, Bardou VJ, Clark GM and Elledge RM: Infiltrating lobular carcinoma of the breast: Tumor characteristics and clinical outcome. Breast Cancer Res 6: R149-R156, 2004.

2. Namikawa T, Kobayashi M and Hanazaki K: An unusual giant duodenal mass lesion. Gastroenterology 148: e5-e6, 2015.

3. Namikawa T and Hanazaki K: Clinicopathological features and treatment outcomes of metastatic tumors in the stomach. Surg Today 44: 1392-1399, 2014.

4. Borst MJ and Ingold JA: Metastatic patterns of invasive lobular versus invasive ductal carcinoma of the breast. Surgery 114: 637-641, discussion 641-642, 1993.

5. Namikawa T, Munekage E, Ogawa M, Oki T, Munekage M, Maeda H, Kitagawa H, Sugimoto T, Kobayashi M and Hanazaki K: Clinical presentation and treatment of gastric metastasis from other malignancies of solid organs. Biomed Rep 7: 159-162, 2017.

6. Taal BG, Peterse $\mathrm{H}$ and Boot $\mathrm{H}$ : Clinical presentation, endoscopic features, and treatment of gastric metastases from breast carcinoma. Cancer 89: 2214-2221, 2000.

7. Miettinen M, McCue PA, Sarlomo-Rikala M, Rys J, Czapiewski P, Wazny K, Langfort R, Waloszczyk P, Biernat W, Lasota J, et al: GATA3: a multispecific but potentially useful marker in surgical pathology: a systematic analysis of 2500 epithelial and nonepithelial tumors. Am J Surg Pathol 38: 13-22, 2014.

8. Liu H, Shi J, Wilkerson ML and Lin F: Immunohistochemical evaluation of GATA3 expression in tumors and normal tissues: A useful immunomarker for breast and urothelial carcinomas. Am J Clin Pathol 138: 57-64, 2012.

9. Yukimoto T, Morisaki T, Komukai S, Yoshida H, Yamaguchi D, Tsuruoka N, Miyahara K, Sakata Y, Shibasaki S, Tsunada S, et al: The palliative effect of endoscopic uncovered self-expandable metallic stent placement versus gastrojejunostomy on malignant gastric outlet obstruction: A pilot study with a retrospective chart review in Saga, Japan. Intern Med 57: 1517-1521, 2018.

10. Shi D, Bao YS and Liu YP: Individualization of metal stents for management of gastric outlet obstruction caused by distal stomach cancer: A prospective study. Gastrointest Endosc 78: 277-284, 2013.

11. Jang SH, Lee H, Min BH, Kim SM, Kim HS, Carriere KC, Min YW, Lee JH and Kim JJ: Palliative gastrojejunostomy versus endoscopic stent placement for gastric outlet obstruction in patients with unresectable gastric cancer: A propensity scorematched analysis. Surg Endosc 31: 4217-4223, 2017. 